\title{
A COLLARING THEOREM FOR CODIMENSION ONE MANIFOLDS
}

\author{
ROBERT J. DAVERMAN AND FRED C. TINSLEY
}

(Communicated by James West)

\begin{abstract}
The chief result implies that an $n$-manifold $S$ embedded in the interior of an $(n+1)$-manifold $M$ as a closed, separating subset is locally flatly embedded if the embedding is well behaved in a locally peripheral sense and if $S$ has arbitrarily close neighborhoods $Q$ such that the fundamental groups of appropriate components of $Q \backslash S$ admit a uniform finite upper bound on the number of generators.
\end{abstract}

Let $M$ denote a compact $(n+1)$-manifold, possibly with boundary, and let $S \subset$ Int $M$ denote a closed $n$-manifold such that $M \backslash S$ has two components $U$ and $U^{\prime}$. The main theorem establishes that $S$ has a collar in $S \cup U$ if the fundamental group of the end of $U$ is finitely generated and $S$ is locally peripherally collared from $U$. As a corollary, $S$ has a collar in $S \cup U$ if $S$ is locally flat except at the points of a Cantor set $C \subset S$ standardly embedded in $S$ and if $U$ is an open collar (that is, $U$ is homeomorphic to the product of $(0,1]$ with another closed $n$-manifold).

Interest in the issue emerged from our constructions [DT1, DT2] of $(n+1)$ manifolds $M$ formed as a union of copies of $S \times[-1,0]$ and $S^{\prime} \times(0,1]$, where $S, S^{\prime}$ are closed $n$-manifolds of different homotopy types and the level corresponding to $S \times 0$ is locally flat modulo a Cantor set. In these constructions one observes that the Cantor set turns out to be wildly embedded in $S \times 0$. Is wildness necessary? The result here gives an affirmative answer, for otherwise a bicollar on $S \times 0$ would force $S$ and $S^{\prime}$ to be homotopically equivalent.

Influential in these developments was Kirby's prototype flatness result [K], which promises that an $n$-sphere $S$ in $\mathbb{R}^{n}(n>3)$ is bicollared if it is locally flat modulo a Cantor set that is twice tame-tame both in $\mathbb{R}^{n}$ and in $S$. Another more direct influence was the paper of Burgess [B], which seems to be the first to combine conditions on local peripheral structure, defined in the next paragraph, with global conditions near the codimension one submanifold, to derive conclusions about local flatness, in the case of [B], for embeddings in 3-manifolds.

Let $M$ be a connected $(n+1)$-manifold, possibly with boundary, $S \subset \operatorname{Int} M$

Received by the editors June 18, 1992.

1991 Mathematics Subject Classification. Primary 57N45, 57N35; Secondary 57N40, 57N70.

Key words and phrases. Codimension one manifold, locally flat embedding, locally peripherally collared, wild Cantor set, open collar neighborhood. 
an $n$-manifold embedded as a closed, separating subset of $M$, and $U$ a component of $M \backslash S$. Then $S$ is locally peripherally collared from $U$ if, for each $\varepsilon>0$ and each $s \in S$, there exists a neighborhood $N_{s}$ of $s$ in $S$ with $\operatorname{diam}\left(N_{s}\right)<\varepsilon$ and $\partial N_{s}$ a simply connected $(n-1)$-manifold that is collared from $U$. The latter means that there exists an embedding $\lambda: \partial N_{s} \times[0,1] \rightarrow \mathrm{Cl}(U)$ with $\partial N_{s}=S \cap$ image $(\lambda)=\lambda\left(\partial N_{s} \times 0\right)$. (Remark: When $n=2$, delete the impossible requirement that $\partial N_{s}$ be 1-connected.)

Furthermore, $S$ is said to have an open collar neighborhood in $U$ if $S$ has a neighborhood $P$ such that $P \cap U$ is topologically equivalent to $S^{\prime} \times(0,1)$, for some closed $n$-manifold $S^{\prime}$.

For completeness, here are definitions of the key older terms. A codimension $k$ submanifold $S$ in the interior of an $m$-manifold $M$ is locally flat at $s \in S$ if $s$ has a neighborhood $U_{s}$ such that $\left(U_{s}, U_{s} \cap S\right)$ is homeomorphic as a pair to $\left(\mathbb{R}^{m}, \mathbb{R}^{m-k}\right)$. A Cantor set $C \subset M$ is tame in $M$ if there is a 1-manifold $S$ such that $C \subset S \subset M$ and $S$ is locally flat at every point $s \in S$.

Local Flatness Theorem. Suppose $M$ is a compact $(n+1)$-manifold with boundary, $n \geq 3, S$ is a component of $\partial M, c: S \times[0,1] \rightarrow S \cup \operatorname{Int} M$ is a collar on $S, \bar{U}=M \backslash$ image $(c)$, and $c(S \times 1)$ is locally peripherally collared from $U$ and has an open collar neighborhood in $U$. Then $c(S \times 1)$ is collared from $U$.

Proof. Assume $c(S \times 1)$ has a neighborhood $P$ such that there is a homeomorphism $\psi$ of $S^{\prime} \times(0,1)$ onto $P \cap U$. We begin by claiming that $c(S \times 1)$ contains at most finitely many points at which $U$ fails to be locally 1 -connected. Fix $\varepsilon>0$, and let $k$ be the number of generators for $\pi_{1}\left(S^{\prime}\right)$. The claim will be proved by showing that, for any set of $k+1$ points $s_{0}, \ldots, s_{k}$ in $c(S \times 1)$, at least one $s_{i}$ has a neighborhood $W_{i}$ such that all loops in $W_{i} \cap U$ are contractible in an $\varepsilon$-subset of $U$.

First, identify neighborhoods $N_{i}$ of $s_{i}$ in $c(S \times 1)$ fulfilling the definition of locally peripherally collared for this choice of $\varepsilon$, subject to the additional requirement that $N_{i} \cap N_{j}=\varnothing$ when $i \neq j$, and let $\lambda_{i}\left(\partial N_{i} \times[0,1]\right)$ denote the boundary collar. Build a neighborhood $Q_{1}$ of $c(S \times 1)$ in $\mathrm{Cl} U$ such that the components $V_{i}$ of $Q_{1} \backslash \bigcup\left(\lambda_{s}\left(\partial N_{s} \times[0,1]\right)\right)$ with $N_{i} \subset \mathrm{Cl} V_{i}(i=0,1, \ldots, k)$ satisfy:

(1) $\operatorname{diam} V_{i}<\varepsilon$,

(2) $V_{i} \cap V_{j}=\varnothing$ when $i \neq j$, and

(3) $Q_{1} \cap \lambda_{i}\left(\partial N_{i} \times[0,1]\right) \cap \mathrm{Cl}\left(V_{i}\right)$ is simply connected.

Second, produce a neighborhood $Q_{2} \subset Q_{1}$ of $c(S \times 1)$ in $\mathrm{Cl} U$ such that $Q_{2} \cap U=\psi\left(S^{\prime} \times(0, r)\right)$.

Third, construct a neighborhood $Q_{3} \subset Q_{2}$ of $c(S \times 1)$ in $\mathrm{Cl} U$ such that the components $W_{i}$ of $Q_{3} \backslash \bigcup\left(\lambda_{s}\left(\partial N_{s} \times[0,1]\right)\right)$ with $N_{i} \subset \mathrm{Cl} W_{i}$ again satisfy:

(3') $Q_{3} \cap \lambda_{i}\left(\partial N_{i} \times[0,1]\right) \cap \mathrm{Cl}\left(W_{i}\right)$ is simply connected.

Analogs of (1) and (2) also hold, simply because $W_{i} \subset V_{i}$ for $i=0,1, \ldots, k$.

Now the crucial observation is that one of the inclusions $U \cap \mathrm{Cl} W_{i} \rightarrow U \cap \mathrm{Cl} V_{i}$ induces a trivial $\pi_{1}$-homomorphism. To check, apply the Seifert-van Kampen Theorem and $\left(3^{\prime}\right)$ to see that $\pi_{1}\left(U \cap Q_{3}\right)$ has a free product decomposition involving factors $\pi_{1}\left(\mathrm{Cl} W_{i}\right)$ and, similarly, $\pi_{1}\left(U \cap Q_{1}\right)$ has a free product decomposition involving factors $\pi_{1}\left(\mathrm{Cl} V_{i}\right)$; furthermore, the inclusion-induced homomorphism $\theta: \pi_{1}\left(U \cap Q_{3}\right) \rightarrow \pi_{1}\left(U \cap Q_{1}\right)$ sends $\pi_{1}\left(\mathrm{Cl} W_{i}\right)$ into $\pi_{1}\left(\mathrm{Cl} V_{i}\right)$. 
Since $\Theta$ factors through $\pi_{1}\left(S^{\prime} \times(0, r)\right)$, image $(\theta)$ can be generated by a set with $k$ elements, and Grushko's Theorem (cf. [M, pp. 224ff]) certifies that at least one of the images $\pi_{1}\left(U \cap \mathrm{Cl} V_{i}\right) \rightarrow \pi_{1}\left(U \cap \mathrm{Cl} W_{i}\right)$ is trivial.

Based on this verification of the initial claim, one has by [Ce, D2] for $n>3$ and $[Q]$ for $n=3$ that $c(S \times 1)$ is locally flat except possibly at the points of some finite set. Finally, since $n+1 \geq 4, c(S \times 1)$ has no isolated points of wildness $[\mathrm{Ca}]$ (or $[\mathrm{K}])$, so it is locally flat everywhere.

The argument actually establishes the following:

Theorem. Suppose $M$ is an $(n+1)$-manifold with boundary, $n \geq 3, S$ is a compact component of $\partial M, c: S \times[0,1] \rightarrow S \cup \operatorname{Int} M$ is a collar on $S$, $U=M \backslash \operatorname{image}(c)$, and $c(S \times 1)$ is locally peripherally collared from $U$. Suppose there exists a positive integer $k$ such that for every neighborhood $Q$ of $c(S \times 1)$ there is another neighborhood $Q^{\prime} \subset Q$ of $c(S \times 1)$ for which the image of the inclusion-induced $\pi_{1}\left(Q^{\prime} \cap U\right) \rightarrow \pi_{1}(Q \cap U)$ is generated by $k$ elements. Then $c(S \times 1)$ is collared from $U$.

Corollary 1. Suppose $M$ is a connected $(n+1)$-manifold, $n \geq 3, S \subset \operatorname{Int} M$ is a compact n-manifold, $M \backslash S$ has two components $U, U^{\prime}$, and $S$ has an open collar neighborhood in $U$. Suppose also $S$ is locally flat except possibly at the points of some Cantor set standardly embedded in $S$. Then $S$ is collared from $U$.

Proof. According to arguments like those in [D3] or [D5], there exists an embedding $e: \mathrm{Cl} U \rightarrow M$ such that $e(S)$ is collared from $M \backslash e(U)$.

When $n=2$ one encounters obvious exceptions to the Local Flatness Theorem. For instance, there is a classical example due to Fox and Artin [FA] of a wild 3-cell $C \subset \mathbb{R}^{3}$ such that $\mathbb{R}^{3} \backslash C$ is homeomorphic to $S^{2} \times(0,1)$, yet $\partial C$ is locally peripherally collared from $\mathbb{R}^{3} \backslash C$, since $\partial C$ is locally flat except at one point. Next we detail a sense in which all exceptions are of this type.

Three-Dimensional Local Flatness Theorem. Suppose $M$ is a compact 3manifold with boundary and $S \subset M$ is a closed, connected 2-manifold such that $M \backslash S$ has components $U, U^{\prime}$, where $S$ is collared from $U^{\prime}$. Suppose $S$ has an open collar neighborhood in $U$ and $S$ is locally peripherally collared from $U$. Then $S$ is locally flat at each point, provided $S$ is not a 2-sphere, in which case $S$ is locally flat except possibly at one point.

Proof. Restrict $M$, if necessary, so $\mathrm{Cl} U^{\prime}=S \times(-1,0]$ and $U \approx S^{\prime} \times(0,1)$. As then $M$ is partitioned into mutually exclusive copies of $S$ and $S^{\prime}$, these two manifolds have the same homology type [D4], which in this dimension implies they are homeomorphic. The conclusions follow from [B, D1].

We close by stating the application to crumpled laminations.

Corollary 2. Suppose $p: M \rightarrow(-1,1)$ is a proper map defined on a connected, orientable $(n+1)$-manifold $M$ such that each $p^{-1} t$ is a closed, orientable $n$ manifold, with $p^{-1} t$ bicollared in $M$ for all $t \neq 0$ and $p^{-1} 0$ locally flat except possibly at the points of some Cantor set standardly embedded in $p^{-1} 0$. Then all pairs of manifolds $p^{-1} s, p^{-1} t$ have the same homotopy type. 


\section{REFERENCES}

[B] C. E. Burgess, Criteria for a 2-sphere in $S^{3}$ to be locally tame modulo two points, Michigan Math. J. 14 (1967), 321-330.

[Ca] J. C. Cantrell, Almost locally flat embeddings of $S^{n-1}$ in $S^{n}$, Bull. Amer. Math. Soc. 69 (1963), 716-718.

[Ce] A. V. Cernavskii, Coincidence of local flatness and local simple-connectedness for embeddings of (n-1)-dimensional manifolds in n-dimensional manifolds when $n>4$, Math. USSR Sb. 91 (1973), 279-296.

[D1] R. J. Daverman, Non-homeomorphic approximation of manifolds with surfaces of bounded genus, Duke Math. J. 37 (1970), 619-625.

[D2] Locally nice codimension one decompositions are locally flat, Bull. Amer. Math. Soc. 79 (1973), 410-413.

[D3] - Every crumpled n-cube is a closed n-cell-complement, Michigan Math. J. 24 (1977), 225-241.

[D4] Decompositions into codimension one submanifolds, Compositio Math. 55 (1985), 185-207.

[D5] - Every crumpled 4-cube is a closed 4-cell-complement, Topology Appl. 26 (1987), 107-113.

[DT1] R. J. Daverman and F. C. Tinsley, Laminations, finitely generated perfect groups, and acyclic mappings, Michigan Math. J. 33 (1986), 343-351.

[DT2] __, A controlled plus construction for crumpled laminations, Trans. Amer. Math. Soc. (to appear).

[FA] R. H. Fox and E. Artin, Some wild cells and spheres in three-dimensional space, Ann. of Math. (2) 49 (1948), 979-990.

[K] R. C. Kirby, On the set of non-locally flat points of a submanifold of codimension one, Ann. of Math. (2) 88 (1968), 281-290.

[M] W. S. Massey, Algebraic topology: an introduction, Springer-Verlag, Berlin, 1977.

[Q] F. Quinn, Ends of maps, III: dimensions 4 and 5, J. Differential Geom. 17 (1982), 503-521. 1300

Department of Mathematics, University of Tennessee, KNoxville, TenNessee 37996-

E-mail address: davermaneutkvx.utk.edu 80903

Department of Mathematics, The Colorado College, Colorado Springs, Colorado

E-mail address: ftinsleyeccnode.colorado.edu 